\title{
Meson screening masses at finite temperature with Highly Improved Staggered Quarks
}

\author{
Yu Maezawa* ${ }^{* \dagger}$ \\ Physics Department, Brookhaven National Laboratory, Upton, NY 11973, USA \\ E-mail: ymaezawa@quark.phy.bnl.gov
}

\section{Alexei Bazavov}

Physics Department, Brookhaven National Laboratory, Upton, NY 11973, USA

\section{Frithjof Karsch}

Physics Department, Brookhaven National Laboratory, Upton, NY 11973, USA

Fakultät für Physik, Universität Bielefeld, D-33615 Bielefeld, Germany

\section{Peter Petreczky}

Physics Department, Brookhaven National Laboratory, Upton, NY 11973, USA

\section{Swagato Mukherjee}

Physics Department, Brookhaven National Laboratory, Upton, NY 11973, USA

\begin{abstract}
We report on the first study of the screening properties of the mesonic excitations with strange $(s)$ and charm $(c)$ quarks, specifically the ground states of the pseudo-scalar and vector meson excitations for the $\bar{s} s, \bar{s} c$ and $\bar{c} c$ flavor combinations, using the Highly Improved Staggered Quark action with dynamical physical strange quark and nearly-physical up and down quarks. By comparing with their respective vacuum meson masses and by investigating the influence of the changing temporal boundary conditions of the valence quarks we study the thermal modifications of these mesonic excitations. While the $\bar{s} s$ states show significant modifications even below the chiral crossover temperature $T_{c}$, the modifications of the open-charm and charmonium like states become visible only for temperatures $T \gtrsim T_{c}$ and $T \gtrsim 1.2 T_{c}$, respectively.
\end{abstract}

31 st International Symposium on Lattice Field Theory LATTICE 2013

July 29-August 3, 2013

Mainz, Germany

\footnotetext{
* Speaker.

†Current address: Fakultät für Physik, Universität Bielefeld, D-33615 Bielefeld, Germany
} 


\section{Introduction}

In-medium properties of hadronic excitations are important for understanding the nature of the Quark Gluon Plasma (QGP) created in the relativistic heavy-ion collision experiments at RHIC and LHC [1]. Inside QGP the interaction between a heavy quark-antiquark pair gets weakened due to the screening effects of the intervening deconfined colored medium. This telltale signature of the presence of a color deconfined medium is expected to be manifested through melting of heavy quarkonium states, bound states of a quark-antiquark pair. In particular, melting of the charmonium states has been proposed as a signal for the creation of the QGP in the heavy-ion collision experiments [2]. Thus it is important to reveal the fates of these meson states at high temperatures.

The lattice simulations enable non-perturbative calculations of physical quantities from firstprinciple QCD. The lattice QCD formalism is based on the Euclidian space-time and mesonic properties, in general, extracted from the corresponding current-current correlation functions along the temporal direction. It is, however, difficult to study in-medium properties of mesons using their temporal correlation functions since at non-zero temperatures the physical distance along the temporal direction is limited by the inverse temperature scale, $1 / T$. To study in-medium properties of the quarkonia from the temporal correlators, several approaches have been proposed, such as the maximum entropy method, the variational technique etc. [3].

Spatial correlation functions of mesonic excitations are free from such a limitation. The spatial correlation functions, $C(z, T)$ enable us to investigate thermal modification of the mesonic excitations at non-zero temperatures, as they too contain information about the temperature dependence of the spectral functions, $\rho(\omega, T)$, of the mesonic excitations [4]

$$
C(z, T)=\int_{0}^{\infty} \frac{2 d \omega}{\omega} \int_{-\infty}^{\infty} d p_{z} e^{i p_{z} z} \rho\left(\omega, p_{z}, T\right) .
$$

At large distances the spatial correlation functions decay exponentially characterized by the decay constant know as the screening mass $(M)$. At small enough temperatures when there exists a welldefined mesonic bound state with $\rho(\omega) \sim \delta\left(\omega^{2}-p_{z}^{2}-m_{0}^{2}\right)$, then $M$ becomes equal to the (pole) mass $m_{0}$ of the meson. On the other hand, at high enough temperatures when the mesonic excitation is completely melted and consists of a free quark-antiquark pair then $M_{\text {free }}(T)=2 \sqrt{(\pi T)^{2}+m_{q}^{2}}$, where $m_{q}$ is the bare mass of the valence quark. Such behavior of the screening mass in the non-interacting limit is the consequence of the fact that at non-zero temperatures while traversing across the temporal boundary the free (anti)quark picks up at least a $\pi T$ contribution from the lowest Matsubara frequency due the anti-periodic temporal boundary condition (APB). Thus the transition between these two limiting values of the screening mass can used as an indicator for the thermal modification and eventual dissolution of the mesonic excitations.

Moreover, if a valence quark-antiquark pair exists as a mesonic bound state then due to the bosonic nature of this excitation its screening mass is not expected to be sensitive to the antiperiodic (fermionic) nature of the temporal boundary condition of its constituent (anti)quark. In such a scenario, even if one imposes a periodic temporal boundary condition (PB) for the quarks the screening mass of the mesonic excitation is expected to remain largely unchanged compared to the usual APB case. On the other hand, for a non-interacting quark-antiquark pair with PB the 
screening mass will be $M_{\text {free }}(T)=2 m_{q}$, instead of the $M_{\text {free }}(T)=2 \sqrt{(\pi T)^{2}+m_{q}^{2}}$ for the case of usual APB. Thus, by studying the influence of the changing temporal boundary condition of the quarks on the screening mass of the quark-antiquark system we can get additional information on the possible existence of mesonic bound states inside the QGP [4].

\section{Simulation details}

Lattice QCD studies of the screening masses have been performed within the quenched approximation [5, 6] and also in full-QCD using the staggered-type quarks [4, 7, 8, 9] as well as using Wilson-type quarks [10]. In this work we report, for the first time, studies of the meson screening masses using the Highly Improved Staggered Quark (HISQ) action with dynamical physical strange quark and nearly-physical up and down quarks. At a given lattice spacing the HISQ action is known to have taste symmetry violations that are smaller than those observed with all other staggered-type actions currently are used in studies of lattice QCD thermodynamics [11]. In this work we study the spatial correlation functions of the mesonic excitations with strange $(s)$ and charm $(c)$ quarks, specifically the ground states of the pseudo-scalar and vector meson excitations for the $\bar{s} s, \bar{s} c$ and $\bar{c} c$ flavor combinations.

We calculate the meson correlation functions on gauge configurations generated with $2+1$ flavor QCD using the HISQ action [11]. The strange quark mass $m_{s}$ is adjusted to its physical value and the light quark masses are fixed at $m_{l}=m_{s} / 20$, corresponding to $m_{\pi} \simeq 160 \mathrm{MeV}$ and $m_{K} \simeq 504$ $\mathrm{MeV}$ at zero temperature. Charm quarks are introduced only as valance quarks. The valence charm quark mass is adjusted to reproduce a spin-averaged charmonium mass, $\frac{1}{4}\left(m_{\eta_{c}}+3 m_{J / \psi}\right)$, at zero temperature. The majority of the calculations are performed using $N_{\tau}=12$ lattices with the lattice couplings of $\beta=6.664-7.280$ which corresponds to temperatures $T=138-245 \mathrm{MeV}$. For $N_{\tau}=10,8,6,4$ lattices we adopt the fixed-scale approach by performing calculations at a fixed $\beta=7.280$ with temperatures ranging from $T=297-743 \mathrm{MeV}$. For all calculations the aspect ratios of the lattices are chosen to be $N_{s} / N_{\tau}=4$. For the chiral crossover temperature we use the continuum extrapolated value of $T_{c}=154 \pm 9 \mathrm{MeV}$ [11].

In the staggered formulation a quark contains four valence tastes and meson operators are defined as $\mathscr{M}=\bar{q}\left(\Gamma^{D} \times \Gamma^{F}\right) q, \Gamma^{D}$ and $\Gamma^{F}$ being products of the Dirac Gamma matrices which generate spin and taste structures, respectively [12]. In this study, we focus only on local meson operators with $\Gamma^{D}=\Gamma^{F}=\Gamma$. By using staggered quark fields $\chi(x)$ at $\boldsymbol{x}=(x, y, z, \tau)$ the meson operators can be written as $\mathscr{M}(\boldsymbol{x})=\tilde{\phi}(\boldsymbol{x}) \bar{\chi}(\boldsymbol{x}) \chi(\boldsymbol{x})$, where $\tilde{\phi}(\boldsymbol{x})$ is a phase factor depending on the choice of $\Gamma$. We calculate only the quark-line connected part of the meson correlators since the effect due to the disconnected part is expected to be small. The spatial correlation function along the $z$ direction, projected to zero transverse momentum, is obtained as

$$
C(z)=\sum_{x, y, \tau} \phi(x)\left\langle\left(M_{0 x}^{-1}\right)^{\dagger} M_{0 x}^{-1}\right\rangle
$$

where $M_{0 x}^{-1}$ is the staggered fermion propagator from $\mathbf{0}$ to $\boldsymbol{x}$ and $\phi(\boldsymbol{x})$ is the phase factor defined as $\phi(\boldsymbol{x})=-(-1)^{x+y+t} \tilde{\boldsymbol{\phi}}(\boldsymbol{x})$. Since a staggered meson correlator contains two different mesons with 

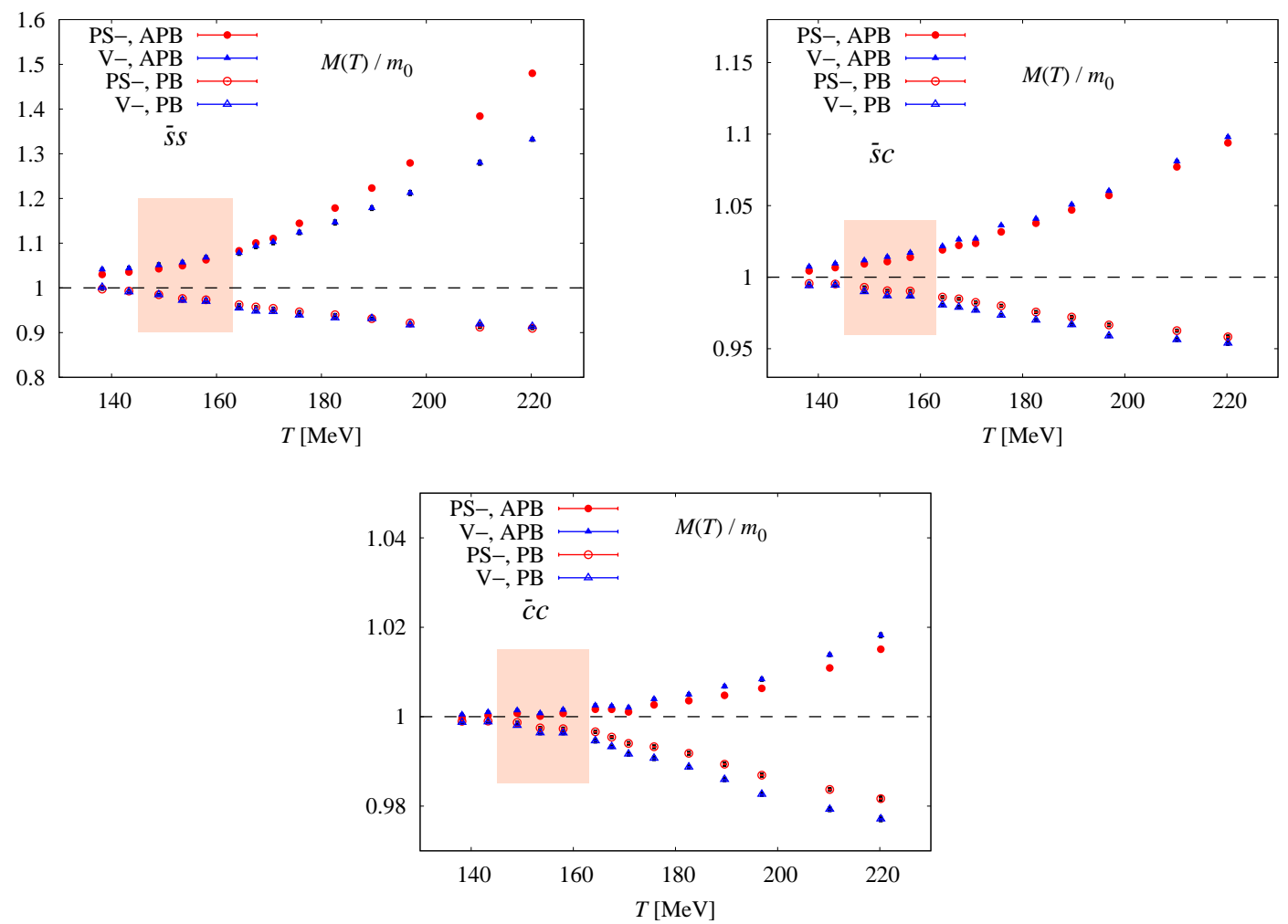

Figure 1: Ratios of the screening masses to the corresponding zero temperature masses for the $\bar{s}$ (top-left), $\bar{s} c$ (top-right) and $\bar{c} c$ (bottom) sectors.

opposite parities, at large distances the lattice correlator can be parametrized as

$$
C(z)=A_{\mathrm{NO}} \cosh \left[M_{-}\left(z-\frac{N_{z}}{2}\right)\right]-(-1)^{z} A_{\mathrm{O}} \cosh \left[M_{+}\left(z-\frac{N_{z}}{2}\right)\right],
$$

where the first (second) term in the right-hand-side characterizes a non-oscillating (oscillating) contribution governed by a negative (positive) parity state. Since at non-zero temperatures the rotational symmetry of the spatial correlations functions reduces to $O(2) \times Z(2)$, the vector meson states must be decomposed into transverse and longitudinal components. Here we only focus on the meson screening masses in the pseudo-scalar $(\mathrm{PS}-)$ and vector-transverse $(\mathrm{V}-)$ channels with the negative parity, defined by $(\Gamma, \phi)=\left(\gamma_{5}, 1\right)$ and $\left(\gamma_{1,2},(-1)^{x}\right.$ and $\left.(-1)^{y}\right)$, respectively. At zero temperature, these correspond to $(\mathrm{PS}-, \mathrm{V}-)=\left(\eta_{\bar{s} s}, \phi\right),\left(D_{s}, D_{s}^{*}\right),\left(\eta_{c}, J / \psi\right)$ for the $\bar{s} s, \bar{s} c$ and $\bar{c} c$, respectively, where $\eta_{\bar{s} s}$ is an unphysical state with the mass $M_{\eta_{\overline{s s}}}=\sqrt{2 M_{K}^{2}-M_{\pi}^{2}}$. We investigate thermal properties of these states by studying temperature dependence of the corresponding screening masses using both periodic and anti-periodic temporal boundary conditions for the valence quarks.

\section{Results}

We have calculated the spatial meson correlators for the $\bar{s} s, \bar{s} c$ and $\bar{c} c$ flavor combinations and 

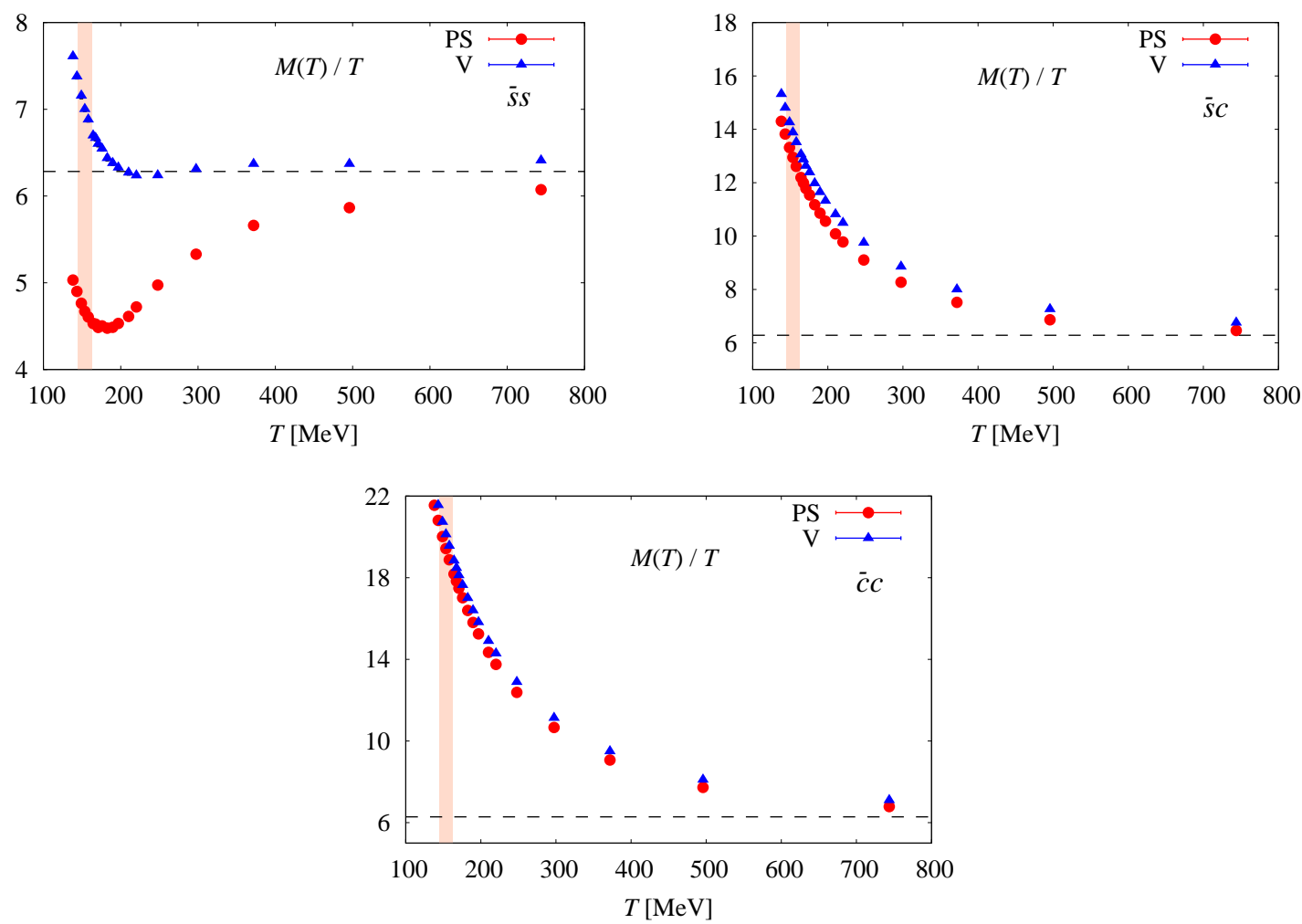

Figure 2: High temperature behavior of the screening masses, in units of temperature, for the $\bar{s}$ (top-left), $\bar{s} c$ (top-right) and $\bar{c} c$ (bottom) sectors.

extracted the screening masses of the ground states in pseudo-scalar (PS-) and vector (V-) channels by fitting the correlators at large distances with Eq. (2.2) using Bayesian fits ${ }^{1}$ [13]. Statistical errors have been estimated using bootstrap analysis.

Figure 1 shows the temperature dependence of the screening masses normalized by the corresponding meson (pole) masses at zero temperature, i.e. $M(T) / m_{0}$, for the $\bar{s} s$ (top-left), $\bar{s} c$ (top-right) and $\bar{c} c$ (bottom) sectors. Circles (triangles) indicate results for the PS - and V-channels, and the filled (open) symbols indicate results obtained with APB (PB) temporal boundary conditions for the valence quarks. The band denotes the chiral crossover temperature. The screening masses agree with the corresponding $T=0$ meson masses at low temperatures. Deviations between APB and $\mathrm{PB}$ conditions gradually set in as the temperature increases. At high temperatures, the screening masses with APB (PB) condition increase (decrease) which is consistent with the behavior in the free limit, i.e. $M_{\text {free }}(T)=2 \sqrt{(\pi T)^{2}+m_{q}^{2}}$ for APB and $2 m_{q}$ for PB, respectively. While the screening masses in the $\bar{s}$ sector (top-left) significantly differ from the corresponding $T=0$ meson masses even for $T<T_{c}$ similar deviations for the $\bar{s} c$ (top-right) and $\bar{c} c$ (bottom) sectors start to appear around $T \gtrsim T_{c}$ and $T \gtrsim 1.2 T_{c}$, respectively. This may imply that the $\eta_{\bar{s} s}$ mesons receive significant thermal modifications even below $T_{c}$ but the $\eta_{c}$ and $J / \psi$ remain largely unaffected by the thermal medium up to $T \lesssim 1.2 T_{c}$.

\footnotetext{
${ }^{1}$ We impose $A_{\mathrm{O}}=0$ on the PS- correlators in $\bar{s}$ and $\bar{c} c$
} 
Figure 2 shows high temperature behaviors of the screening masses, normalized by temperature $M(T) / T$, with APB condition for the $\bar{s}$ (top-left), $\bar{s} c$ (top-right) and $\bar{c} c$ (bottom) sectors. The dashed lines denote the value $2 \pi$ to which the screening masses converge in the high temperature limit of the screening masses for massless non-interacting quarks. The $\bar{s} c$ and $\bar{c} c$ screening masses monotonically decrease with temperature and tend to converge to the massless non-interacting limit of $2 \pi$ for $T \gtrsim 700 \mathrm{MeV}$. On the other hand, the $\bar{s} s$ sector shows more interesting features: the screening masses decrease at low temperature, start to increase again for $T \gtrsim T_{c}$ and then tend to converge towards $2 \pi T$ for $T \gtrsim 700 \mathrm{MeV}$ with the PS $-(\mathrm{V}-)$ channel approaching from below (above).

\section{Summary}

In this work we have investigated the screening masses of the ground state pseudo-scalar and vector mesonic excitations for the $\bar{s} s, \bar{s} c$ and $\bar{c} c$ flavor combinations. Calculations have been performed using $2+1$ flavors of dynamical HISQ along with valence charm quarks. We have investigated the signatures of thermal modifications of these states by comparing their screening masses with the corresponding zero-temperature meson masses. We have also studied the influence of the changing temporal boundary conditions of the valence quarks on the screening masses of these states. Combining these studies we have found clear signatures of thermal modifications for the $\bar{s} s$ states even for $T<T_{c}, T_{c}=154 \mathrm{MeV}$ being the chiral crossover temperature. For the $\bar{s} c$ and $\bar{c} c$ states distinctive signatures of thermal modifications appear only for $T \gtrsim T_{c}$ and $T \gtrsim 1.2 T_{c}$, respectively. On the other hand, for all sectors the screening masses approach the non-interacting massless quark gas values of $2 \pi T$ only at very high temperatures of $T \gtrsim 700 \mathrm{MeV}$.

\section{Acknowledgement}

Numerical calculations were carried out on the USQCD Clusters at the Jefferson Laboratory, USA and the NYBlue at the Brookhaven National Laboratory, USA. This work was partly supported by through the Contract No. DE-AC02-98CH10886 with the U.S. Department of Energy. Partial support for this work was also provided through Scientific Discovery through Advanced Computing (SciDAC) program funded by U.S. Department of Energy, Office of Science, Advanced Scientific Computing Research (and Basic Energy Sciences/Biological and Environmental Research/High Energy Physics/Fusion Energy Sciences/Nuclear Physics).

\section{References}

[1] H. Satz, Extreme states of matter in strong interaction physics. An introduction, Lect. Notes Phys. 841, 1 (2012).

[2] T. Matsui and H. Satz, J/ $\psi$ Suppression by Quark-Gluon Plasma Formation, Phys. Lett. B 178, 416 (1986).

[3] See recent review and references therein; A. Mocsy, P. Petreczky and M. Strickland, Quarkonia in the Quark Gluon Plasma, Int. J. Mod. Phys. A 28, 1340012 (2013). 
[4] F. Karsch, E. Laermann, S. Mukherjee and P. Petreczky, Signatures of charmonium modification in spatial correlation functions, Phys. Rev. D 85, 114501 (2012).

[5] C. E. Detar and J. B. Kogut, Measuring the Hadronic Spectrum of the Quark Plasma, Phys. Rev. D 36, 2828 (1987).

[6] P. de Forcrand et al. [QCD-TARO Collaboration], Meson correlators in finite temperature lattice QCD, Phys. Rev. D 63, 054501 (2001).

[7] G. Boyd, S. Gupta, F. Karsch and E. Laermann, Spatial and temporal hadron correlators below and above the chiral phase transition, Z. Phys. C 64, 331 (1994).

[8] I. Pushkina et al. [QCD-TARO Collaboration], Properties of hadron screening masses at finite baryonic density, Phys. Lett. B 609, 265 (2005).

[9] M. Cheng et al., Meson screening masses from lattice QCD with two light and the strange quark, Eur. Phys. J. C 71, 1564 (2011).

[10] H. Iida, Y. Maezawa and K. Yazaki, Hadron properties at finite temperature and density with two-flavor Wilson fermions, PoS LATTICE 2010, 189 (2010).

[11] A. Bazavov et al. [HotQCD Collaboration], The chiral and deconfinement aspects of the QCD transition, Phys. Rev. D 85, 054503 (2012).

[12] R. Altmeyer et al. [MT(c) Collaboration], The Hadron spectrum in QCD with dynamical staggered fermions, Nucl. Phys. B 389, 445 (1993).

[13] G. P. Lepage, B. Clark, C. T. H. Davies, K. Hornbostel, P. B. Mackenzie, C. Morningstar and H. Trottier, Constrained curve fitting, Nucl. Phys. Proc. Suppl. 106, 12 (2002). 